\title{
Enhanced Antibacterial Effects of Clove Essential Oil by Nanoemulsion
}

\author{
Md Khalid Anwer ${ }^{1}$, Shahid Jamil ${ }^{1}$, Elmutasim Osman Ibnouf ${ }^{2}$ and Faiyaz Shakeel ${ }^{3 *}$ \\ ${ }^{1}$ Department of Pharmaceutics, College of Pharmacy, Salman Bin Abdulaziz University, Al-Kharj, Saudi Arabia \\ ${ }^{2}$ Department of Pharmaceutical Microbiology, College of Pharmacy, Salman Bin Abdulaziz University, Al-Kharj, Saudi Arabia \\ ${ }^{3}$ Center of Excellence in Biotechnology Research (CEBR), King Saud University, P.O. Box 2460, Riyadh 11451, Saudi Arabia
}

\begin{abstract}
The aim of present study was to develop and evaluate nanoemulsion formulations of clove essential oil (CEO) for its antibacterial effects in comparison with pure CEO and standard amikacin antibiotic (positive control). Different nanoemulsions of CEO were developed by aqueous phase titration method via construction of pseudo-ternary phase diagrams and investigated for thermodynamic stability and self-nanoemulsification tests. Selected formulations (F1-F5) were characterized for droplet size distribution, viscosity, zeta potential, transmittance and surface morphology. Based on lowest droplet size (29.1 $\mathrm{nm})$, lowest PI (0.026), lowest viscosity (34.6 cp), optimal zeta potential (-31.4 mV), highest transmittance $(99.4 \%)$ and lowest concentration of Triacetin $(8 \%$ w/w), CEO nanoemulsion F1 (containing $1 \% \mathrm{w} / \mathrm{w}$ of CEO, $8 \%$ w/w of Triacetin, $15 \%$ w/w of Tween-80, $15 \%$ w/w of Labrasol and $61 \%$ w/w of water) was subjected to antibacterial studies in comparison with pure oil and standard amikacin. The antibacterial effects of $F 1$ were found to be superior over pure oil against all bacterial strains investigated. However, the antibacterial effects of $\mathrm{F} 1$ were highly comparable with standard amikacin against all bacterial strains. The minimum inhibitory concentrations (MICs) of F1 were observed in the range of 0.075$0.300 \% \mathrm{w} / \mathrm{w}$ as compared to pure oil (MICs 0.130-0.500 \% w/w) and standard amikacin (MICs 2-16 $\mu \mathrm{g} / \mathrm{ml}$ ). These results indicated the potential of nanoemulsions for enhancing the therapeutic efficacy of natural bioactive ingredients such as CEO.
\end{abstract}

Key words: amikacin, antibacterial effects, clove oil, droplet size, nanoemulsion

\section{INTRODUCTION}

In the recent years, essential oils have gained massive acceptance due to their applications in number of pharmaceutical and food products because of their potential in inhibiting the growth and propagations of a wide range of microorganisms such as bacteria, fungi and virus ${ }^{1-3)}$. Clove essential oil (CEO) is an essential oil isolated from flower buds of Syzygium aromaticum L. (Family: Myrtaceae), advocated in the treatment of various diseases ${ }^{2-4)}$. Many pharmacological bioactivities including anti-inflammatory, analgesic, antibacterial, antifungal, anti-allergic, anti-carcinogenic and anti-mutagenic activities have been reported for $\mathrm{CEO}^{1,5-10)}$. Several phytochemicals have been identified in CEO such as eugenol, acetyleugenol, isoeugenol, methyleugenol, carvacrol, thymol, cinnamaldehyde, eugenyl acetate, $\beta$-caryophyllene, 2 -heptanone, methyl salicylate, $\alpha$-humulene, gallic acid, ellagic acid and oleanolic acid $\operatorname{etc}^{2-4)}$. Eugenol $(88.58 \%)$ is the major constituent of CEO which displays significant anti-oxidant and insecticidal properties $^{2,11,12)}$. Most of the above mentioned biological activities have also been attributed due to the presence of eugenol $^{1-3,8-10)}$. Nanoemulsions are clear, transparent, isotropic and thermodynamically stable solutions of oil, surfactant and cosurfactant with droplet size of less than 100 $\mathrm{nm}^{13-15)}$. In the recent years, these systems have been investigated extensively as novel nanovehicles to enhance solubilization capacity, therapeutic efficacy and bioavailability of various food components, drugs and pharmaceuticals ${ }^{13-21)}$. CEO is immiscible in water (insoluble) and its principal constituent eugenol shows poor oral absorption/ bioavailability in rats ${ }^{22)}$. Some formulation approaches such as transdermal formulation, nanoemulsion, microemulsion, spray formulations and mucoadhesive tablets have been investigated for solubility, dissolution, therapeutic efficacy and bioavailability enhancement in literature ${ }^{23-27)}$. To the best of our knowledge, antibacterial effects of CEO encap-

\footnotetext{
*Correspondence to: Faiyaz Shakeel, Center of Excellence in Biotechnology Research (CEBR), King Saud University, P.O. Box 2460, Riyadh 11451, Saudi Arabia

E-mail: faiyazs@fastmail.fm

Accepted December 17, 2013 (recieved for review December 7, 2013)

Journal of Oleo Science ISSN 1345-8957 print / ISSN 1347-3352 online

http://www.jstage.jst.go.jp/browse/jos/ http://mc.manusriptcentral.com/jjocs
} 
sulated nanoemulsions have not been investigated in literature. Therefore, the aim of this article was to develop, characterize and evaluate nanoemulsion formulations of CEO in order to enhance its antibacterial effects. The antibacterial effects of CEO nanoemulsions were also compared with pure oil and antibiotic amikacin (positive control) against five bacterial strains namely Bacillus subtilis, Staphyloccocus aureus, Proteus vulgaris, Pseudomonas aeruginosa and Klebseilla pneumonia. All these bacteria are known to cause several infectious diseases such as endovascular infection(Staphyloccocus aureus), food poisoning (Bacillus subtilis), urinary tract infections (Proteus vulgaris), pulmonary \& urinary tract infections (Pseudomonas aeruginosa) and Pneumonia (Klebseilla pneumonia $)^{28-31)}$. Most of these bacterial are resistant to various antibiotics, hence there is an urgent need of an alternative therapy for the treatment of the infections caused by these bacteria.

\section{EXPERIMENTAL}

\subsection{Materials}

Clove essential oil (purity 87.5\%) and glycerol triacetate (Triacetin) were purchased from Loba Chemie Pvt. Ltd. (Mumbai, India) and Alpha Chemica (Mumbai, India), respectively. Caprylocaproyl macrogol-8-glyceride(Labrasol) was obtained as a gift sample from Gattefosse (Cedex, France). Amikacin and polyoxyethylene (20) sorbitan monooleate (Tween-80) were purchased from Sigma Aldrich (St. Louis, MO). Amikacin was used as positive control in present study. Bacillus subtilis (ATCC 10400), Staphyloccocus aureus (ATCC 29213), Proteus vulgaris (clinical isolates), Pseudomonas aeruginosa (clinical isolates) and Klebseilla pneumonia (clinical isolates)were obtained from Department of Pharmaceutical Microbiology, Salman Bin Abdulaziz University (Al-Kharj, Saudi Arabia). All other chemicals used were of Pharmaceutical grades.

\subsection{Construction of pseudo-ternary phase diagrams}

In present study, Triacetin, Tween-80 and Labrasol were used as oil phase, surfactant and cosurfactant, respectively for the development of CEO nanoemulsions. However, deionized water used as aqueous phase. For the construction of pseudo-ternary phase diagrams, Surfactant(Tween-80) and cosurfactant (Labrasol) were mixed in mass ratios of 1:0, 1:2, 1:1, 2:1 and 3:1. Triacetin (oil phase) and a particular mass ratio of surfactant to cosurfactant $\left(\mathrm{S}_{\text {mix }}\right)$ were mixed at mass ratios of 1:9 to 9:1. Pseudo-ternary phase diagrams were developed by aqueous phase titration method by titrating the mixture of oil phase and specific $\mathrm{S}_{\text {mix }}$ slowly with deionized water ${ }^{13)}$. The physical state of prepared nanoemulsions was marked on a pseudo-ternary phase diagram with one axis representing the aqueous phase, second oil phase (Triacetin) and third representing the specific mass ratio of surfactant(Tween-80) to cosurfactant (Labrasol).

\subsection{Preparation of CEO nanoemulsions}

From the pseudo-ternary phase diagram, nanoemulsion zones were pointed out and different formulations (with different compositions) from this location were chosen for the preparation of CEO nanoemulsions. Considering pseudo-ternary phase diagrams, maximum nanoemulsion zones were represented by 1:1 mass ratio of Tween-80 to Labrasol in varying proportions. Therefore, different formulations were selected from 1:1 mass ratio of $\mathrm{S}_{\text {mix }}$. Almost entire range of nanoemulsion occurrence in the phase diagram was taken into account and varied oil compositions with minimum surfactant concentration were selected. $1 \% \mathrm{w} / \mathrm{w}$ of CEO was mixed with required amount of Triacetin (oil phase, varied from 8 to $24 \% \mathrm{w} / \mathrm{w})$. The fixed amount of $\mathrm{S}_{\text {mix }}(30 \% \mathrm{w} / \mathrm{w})$ was added to CEO-Triactin mixture and water was added with drop-wise manner till an apparent and clear solution was obtained. The composition of CEO nanoemulsions is listed in Table 1.

\subsection{Thermodynamic stability and self-nanoemulsification tests}

Thermodynamic stability tests on developed CEO nanoemulsions were performed to eliminate unstable formulations and to select stable one. CEO nanoemulsions were

Table 1 Composition ( $\% \mathrm{w} / \mathrm{w})$ of clove essential oil nanoemulsions (F1-F5) prepared using Triacetin, Tween-80, Labrasol and water.

\begin{tabular}{ccccccc}
\hline \multirow{2}{*}{ Code } & \multicolumn{5}{c}{ Formulation composition $(\% \mathrm{w} / \mathrm{w})$} & \multirow{2}{*}{$\mathrm{S}_{\text {mix }}$ ratio } \\
\cline { 2 - 6 } & Clove essential oil & Triacetin & Tween-80 & Labrasol & Water & \\
\hline F1 & 1 & 8 & 15 & 15 & 61 & $1: 1$ \\
F2 & 1 & 12 & 15 & 15 & 57 & $1: 1$ \\
F3 & 1 & 16 & 15 & 15 & 53 & $1: 1$ \\
F4 & 1 & 20 & 15 & 15 & 49 & $1: 1$ \\
F5 & 1 & 24 & 15 & 15 & 45 & $1: 1$ \\
\hline
\end{tabular}




\section{Enhanced Antibacterial Effects of Clove Oil}

subjected to centrifugation at $5000 \mathrm{rpm}$ for $30 \mathrm{~min}$. The formulations which were observed to be stable at centrifugation were subjected to heating and cooling cycles (3 cycles) between 4 and $50^{\circ} \mathrm{C}$ for $48 \mathrm{~h}$. The formulations which were observed to be stable at heating and cooling cycles were subjected to freeze-pump-thaw cycles (3 cycles) between -21 and $+25^{\circ} \mathrm{C}$ for $24 \mathrm{~h}^{14,15)}$.

However, the objective of self-nanoemulsification test was to investigate any precipitation or phase separation upon dilution with water. To perform this test, $1 \mathrm{ml}$ of each CEO nanoemulsion(F1-F5) was diluted with $500 \mathrm{ml}$ of deionized water. The efficiency of each nanoemulsion was investigated visually using the following grading systems ${ }^{32,33)}$ :

Grade A: Rapid forming clear/transparent nanoemulsion (emulsify within 1 minute)

Grade B: Rapid forming bluish white nanoemulsion (emulsify within 2 minute)

Grade C: Milky emulsions (more than 2 minutes to emulsify)

Grade D: Dull, grayish milky emulsions (more than 3 minutes to emulsify)

Grade E: Formulations with oil globules at the surface

\subsection{Measurement of droplet size, polydispersity and zeta potential}

The mean droplet size, polydispersity index (PI) and zeta potential (ZP) of CEO nanoemulsions (F1-F5) were measured using Malvern Particle Size Analyzer (Malvern Instruments Ltd., Holtsville, NY) at $25^{\circ} \mathrm{C}$. In order to perform these measurements, each nanoemulsion was diluted 200 times with deionized water, sonicated for $10 \mathrm{~min}$ and filtered via $0.45 \mu \mathrm{m}$ membrane filters. The scattering angle for the measurement was fixed at $90^{\circ}$. The scattering signals were detected by a high-sensitivity avalanche photodiode detector ${ }^{34)}$.

\subsection{Measurement of viscosity and transmittance}

The viscosity of CEO nanoemulsions (F1-F5) was measured using Brookfield Viscometer(Brookfield Engineering Laboratories, Middleboro, MA) at $25 \pm 1^{\circ} \mathrm{C}$ without any dilution. However the percentage of transmittance (\% T) of CEO nanoemulsions was measured spectrophotometrically at $550 \mathrm{~nm}$ as reported in literature ${ }^{32,33)}$.

\subsection{Transmission electron microscopy}

Transmission electron microscopy (TEM) operating at 200 KV (Tecnai TF20, Hillsboro, OR) was performed to investigate surface morphology and droplet size distribution of optimized formulation F1. In order to carry out TEM experiments, optimized CEO nanoemulsion (F1) was diluted 200 times with water and a drop was deposited on the carbon-coated copper grid. The excess amount of liquid was blotted and negative staining was performed with $2 \%$ phosphotungstic acid for $10 \mathrm{sec}$ and blotted dry for $30 \mathrm{sec}$.
The grid was then observed under light microscopy operating at $200 \mathrm{KV}^{13)}$.

\subsection{Antibacterial studies}

Based on lowest droplet size, lowest PI, lowest viscosity, optimal ZP, highest\% $\mathrm{T}$ and after all lowest concentration of Triacetin, CEO nanoemulsion F1 was optimized and selected for antibacterial studies as a test formulation. Agarcup diffusion method was used to evaluate antibacterial effects $^{3)}$ in order to compare antibacterial effects of optimized test formulation F1 with standard amikacin which was used as positive control in present study. These studies were carried out against five bacterial species which are known to pathogenic for human and animals namely Bacillus subtilis, Staphyloccocus aureus, Proteus vulgaris, Pseudomonas aeruginosa and Klebseilla pneumonia, ${ }^{38}$. All these bacterial strains were activated in nutrient agar at $37^{\circ} \mathrm{C}$ for $24 \mathrm{~h}$. The selection of these bacterial strains was based on their pharmacological and clinical relevance ${ }^{2,3,28)}$. All bacterial species were cultivated on Mueller-Hinton agar (Oxoid, England). On solidification, $5 \mathrm{~mm}$ holes were made with sterile borer and $0.25 \mathrm{ml}$ of test strains were inoculated in the media separately. The solutions $(100 \mu \mathrm{l})$ of test formulation and amikacin (positive control) were introduced into each well and plates were incubated at $37^{\circ} \mathrm{C}$ for 24 h. Pure clove oil was diluted with sterile normal saline solution $(0.9 \% \mathrm{w} / \mathrm{v})$ with the help of Tween-80 at final concentration of $1 \% \mathrm{w} / \mathrm{w}$ was also applied to evaluate its antibacterial effects. Sterile normal saline solution was used as negative control. Optimized formulation F1 without CEO (blank formulation) was also applied to investigate formulation effects on antibacterial activity. The inhibition zones $(\mathrm{mm})$ around the wells were measured for all sample matrices investigated. The inhibition zones of blank formulation were subtracted from the inhibition zones of CEO nanoemulsions F1. The minimum inhibitory concentration (MIC) of F1, amikacin (positive control) and CEO were also measured by the broth two-fold macro dilution method in Mueller-Hinton agar medium against all bacterial strains ${ }^{3)}$.

\subsection{Statistical analysis}

The significance of difference in physicochemical parameters and antibacterial activities was evaluated by applying the one-way analysis of variance (ANOVA) test using a statistical software package Graphpad Prism(version 4, San Diego, CA). Differences between related parameters were considered statistically significant at $p<0.05$.

\section{RESULTS}

Pseudo-ternary phase diagrams for the development of CEO nanoemulsions were constructed separately for each $\mathrm{S}_{\text {mix }}$ ratio in order to optimize effective formulations (Fig. 


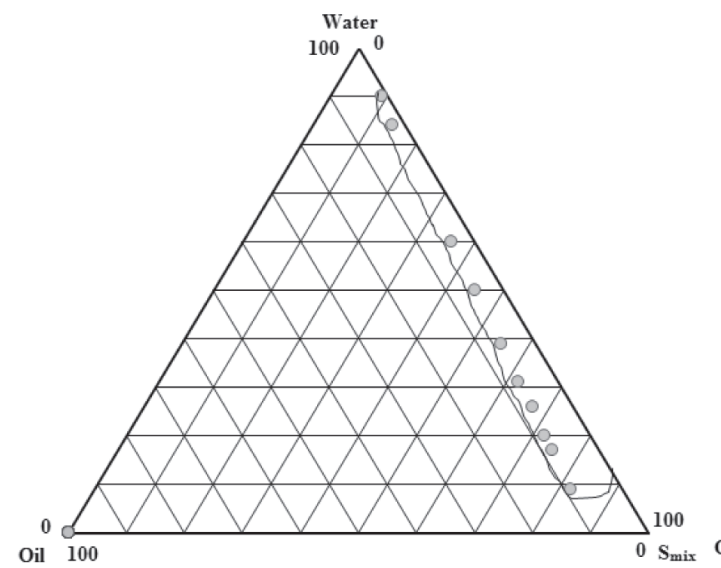

(A)

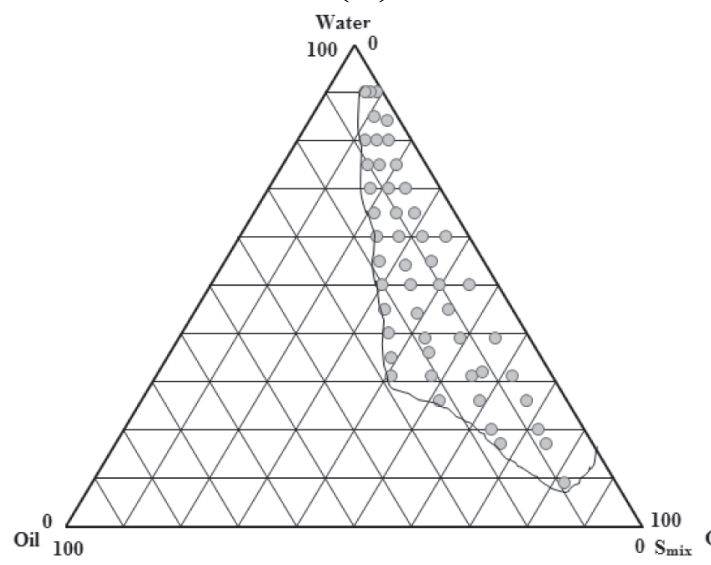

(C)

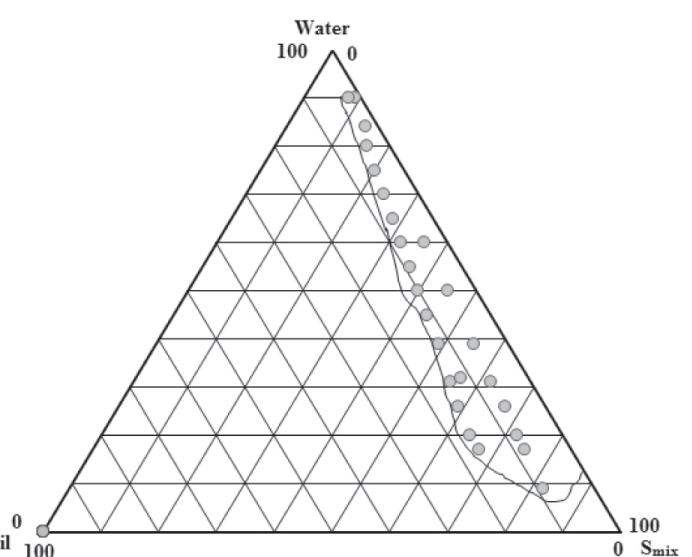

(B)

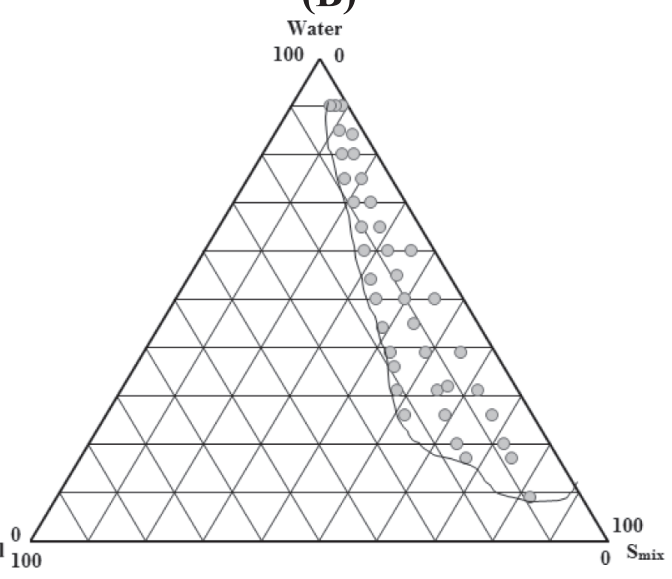

(D)

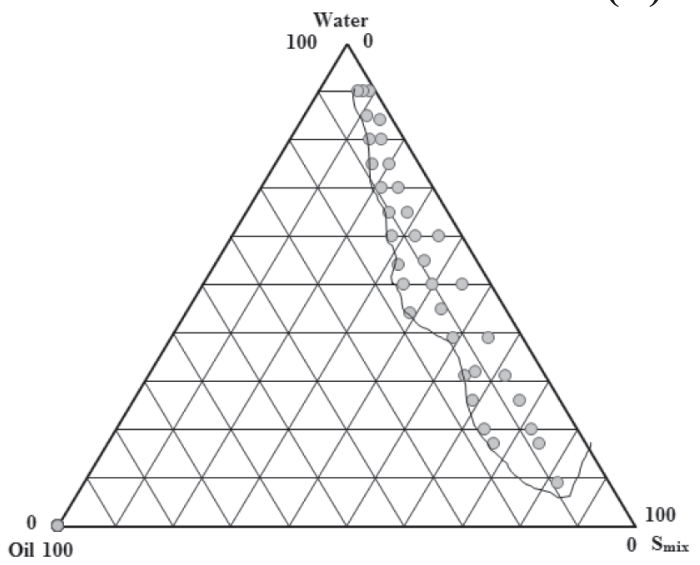

(E)

Fig. 1 Pseudo-ternary phase diagrams showing nanoemulsion zones for oil phase (Triacetin), aqueous phase (water), surfactant (Tween-80) and cosurfactant (Labrsol) at $S_{\text {mix }}$ ratios of A. 1:0, B. 1:2, C. 1:1, D. 2:1 and E. 3:1.

1). From the phase diagrams, it was observed that $S_{\text {mix }} 1: 0$ had very low nanoemulsion zones (Fig. 1A). The highest amount of Triacetin (oil phase) that was solubilized by 1:0 ratio was $9 \% \mathrm{w} / \mathrm{w}$ by utilizing higher percentage of $\mathrm{S}_{\text {mix }}$ $(82 \% \mathrm{w} / \mathrm{w})$. However, in case of $\mathrm{S}_{\text {mix }} 1: 2($ Fig. 1B), when cosurfactant (Labrasol) was used along with the surfactant (Tween-80), the nanoemulsion zones were found to be in- creased rapidly as compared to 1:0 ratio. The highest amount of Triacetin that was solubilized by this ratio was found to be $16 \% \mathrm{w} / \mathrm{w}$ by incorporating $64 \% \mathrm{w} / \mathrm{w}$ of $\mathrm{S}_{\text {mix }}$. On the other hand, when $S_{\text {mix }}$ ratio of 1:1 (Fig. 1C) was studied, the nanoemulsion zones were found to be increased significantly as compared to $1: 0$ and 1:2 ratios. The highest amount of Triacetin that was solubilized by 1:1 ratio was 
found to be $28 \% \mathrm{w} / \mathrm{w}$ by incorporating $41 \% \mathrm{w} / \mathrm{w}$ of $\mathrm{S}_{\text {mix }}$, itself showed the dominance of cosurfactant (Labrasol) along with surfactant (Tween-80) in nanosizing of nanoemulsions. The increased nanoemulsion zones in 1:1 mass ratio of $S_{\text {mix }}$ were probably due to the synergistic effects of Tween-80 and Labrasol. When $\mathrm{S}_{\text {mix }}$ ratio of 2:1 was studied (Fig. 1D), the nanoemulsion zones were started to decreased as compared to 1:1 ratio. The highest amount of Triacetin that was solubilized by this ratio was found to be $22 \% \mathrm{w} / \mathrm{w}$ by incorporation of $52 \% \mathrm{w} / \mathrm{w}$ of $\mathrm{S}_{\text {mix }}$. When $\mathrm{S}_{\text {mix }}$ ratio of $3: 1$ was studied (Fig. 1E), the nanoemulsion zones were found to be decreased further as compared to 2:1 as well as 1:1 ratios. The highest amount of Triacetin that was solubilized by this ratio was found to be $17 \% \mathrm{w} / \mathrm{w}$ by incorporation of $39 \% \mathrm{w} / \mathrm{w}$ of $\mathrm{S}_{\text {mix }}$. For the development and optimization of robust nanoemulsions, the selected formulations were subjected to thermodynamic stability tests in terms of centrifugation, heating \& cooling cycles and freeze-pump-thaw cycles. Thermodynamically stable nanoemulsions (F1-F5) were further investigated for self-nanoemulsification test using A-E grading systems. It was observed that formulations F1, F2 and F3 passed this test with grade A while the formulations F4 and F5 passed this test with grade $\mathrm{B}$.

The results of droplet size distribution, $\mathrm{PI}$ and $\mathrm{ZP}$ of CEO nanoemulsions are summarized in Table 2. The mean droplet size of nanoemulsions (F1-F5) was observed in the range of 29.1-136.9 nm. The droplet size of formulations F1 and F2 was observed to be very close with each other (Table 2). In general, the droplet size was found to enhanced by increasing the concentration of Triacetin (oil phase) in the formulations. The PI of CEO nanoemulsions (F1-F5) was observed in the range of 0.026-1.032. The PI of formulations F1, F2 and F3 was very low (0.026-0.042), indicating excellent uniformity in droplet size distribution. However, the PI of formulations F4 and F5 was very high (0.942-1.032), indicating heterogeneity of droplet size dis- tribution.

The ZP values of CEO nanoemulsions (F1-F5) were observed in the range of -48.6 to $-31.4 \mathrm{mV}$ (Table 2 ). The lowest ZP was observed in formulation F5 $(-48.6 \mathrm{mV})$. However, the highest one was observed in formulation F1 $(-31.4 \mathrm{mV})$.

The viscosity of CEO nanoemulsions (F1-F5) was observed in the range of 34.6-71.3 cp (Table 2). The viscosity of formulations was also found to be enhanced by increasing the concentration of Triacetin in formulations. The $\% \mathrm{~T}$ of CEO nanoemulsions (F1-F5) was observed in the range of $92.3-99.4 \%$ (Table 2). The $\% \mathrm{~T}$ of formulations was also found to be reduced by increasing the concentration of Triacetin in formulations. The highest $\% \mathrm{~T}$ was observed in formulation F1 $(99.4 \pm 0.1 \%)$. However, the lowest one was observed in formulation F5 $(92.3 \pm 0.4 \%)$. The results of $\%$ $\mathrm{T}$ studies indicated transparent behavior of all formulations.

The surface morphology and shape of droplets was studied using TEM. Photomicrograph of optimized nanoemulsion F1 was taken and interpreted for surface morphology and droplet size distribution. The size of all droplets was observed less than $35 \mathrm{~nm}$ as shown in Fig. 2.

The results of antibacterial effects of test, positive control and pure CEO in terms of zone of inhibitions (mm) and MICs are listed in Table 3.

\section{DISCUSSION}

With regard to pseudo-ternary phase diagrams, highest nanoemulsion zones were exposed by $\mathrm{S}_{\text {mix }}$ ratio of 1:1 (Fig. 1C). Therefore, different nanoemulsions with different compositions were precisely selected from Fig. 1 based on evidence based performance of thermodynamic stability studies. Almost the entire region of nanoemulsion occurrence in the phase diagrams was covered and different oil compositions $(8,12,16$ and $20 \% \mathrm{w} / \mathrm{w})$ with fixed surfactant

Table 2 Physicochemical characterization of CEO nanoemulsions (F1-F5) in terms of droplet size, polydispersity, viscosity, zeta potential and transmittance.

\begin{tabular}{cccccc}
\hline \multirow{2}{*}{ Code } & \multicolumn{5}{c}{ Characterization parameters } \\
\cline { 2 - 6 } & $\Delta d m^{*} \pm \mathrm{SD}(\mathrm{nm})$ & $p_{i}{ }^{*}$ & $\eta \pm \mathrm{SD}(\mathrm{cp})$ & $\% T \pm S D$ & $Z P(\mathrm{mV})$ \\
\hline F1 & $29.1 \pm 3.2$ & 0.026 & $34.6 \pm 2.9$ & $99.4 \pm 0.1$ & -31.4 \\
F2 & $31.3 \pm 4.0$ & 0.038 & $41.3 \pm 3.7$ & $99.0 \pm 0.2$ & -32.0 \\
F3 & $127.4 \pm 4.7$ & 0.042 & $64.6 \pm 4.8$ & $98.3 \pm 0.3$ & -38.2 \\
F4 & $134.6 \pm 5.3$ & 1.032 & $68.2 \pm 5.3$ & $95.2 \pm 0.2$ & -42.6 \\
F5 & $136.9 \pm 11.2$ & 0.942 & $71.3 \pm 5.7$ & $92.3 \pm 0.4$ & -48.6 \\
\hline
\end{tabular}

\#mean droplet diameter $(\Delta d m)$, * polydispersity index $\left(p_{i}\right)$, viscosity mean $(\eta), \% T(\%$ transmittance), zeta potential (ZP), standard deviation (SD) 


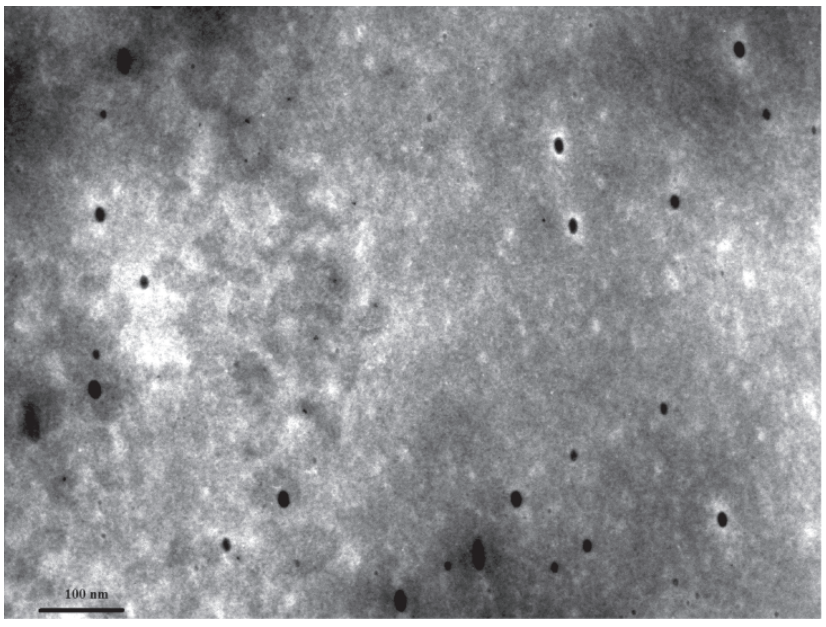

Fig. 2 Transmission electron microscopic (TEM) image of optimized nanoemulsion (F1) showing non-spherical droplets within submicrone range.

concentration $(30 \% \mathrm{w} / \mathrm{w})$ were precisely selected. 1\% w/w of CEO was directly loaded on selected formulations. The composition of these nanoemulsions is listed in Table 1. The objective of these tests was to eliminate metastable and unstable formulations. Only those formulations, which were observed to be stable at all stress conditions of thermodynamic stability tests were selected for self-nanoemulsification test. Self-nanoemulsification tests were performed to investigate the phase separation or drug precipitation upon dilution with water. Largest droplet size was observed in formulation F5 $(136.9 \pm 11.2 \mathrm{~nm})$ due to the presence of highest concentration of Triacetin $(24 \% \mathrm{w} /$ w). However, the droplet size of formulation F1 was found to be lowest $(29.1 \pm 3.2 \mathrm{~nm})$ due to the presence of lowest concentration of Triacetin. Formulation F1 showed least PI value (0.026) suggesting highest uniformity in droplet size distribution. However, highest PI value was observed in formulation F5 (1.032). It has been reported that ZP values in the magnitude of $30 \mathrm{mV}$ in either charge (positive or negative) characterizes a stable formulation ${ }^{32,33}$. In present study, the ZP of formulations F1-F3 was very close to 30 $\mathrm{mV}$, indicating stable formation of F1-F3. However, the ZP of formulations F4 and F5 was deviated from $30 \mathrm{mV}$, indicating unstable formation of F4 and F5. The negative net charge on ZP for all CEO nanoemulsions was probably due to the presence of fatty acid esters in Triacetin in all formulations. The lowest viscosity was observed in formulation $\mathrm{F} 1(34.6 \pm 2.9 \mathrm{cp})$ which was due to its lowest droplet size and the presence of lowest concentration of Triacetin. However, the highest viscosity was observed in formulation F5 $(71.3 \pm 5.7 \mathrm{cp})$ due to largest droplet size and the presence of highest concentration of Triacetin. TEM studies showed non-spherical shape of nanoemulsion droplets which was probably due to the presence of Triacetin and Tween-80 in the formulation. The droplet size distribution recorded by TEM was in good agreement with droplet size distribution recorded by Malvern Particle Size Analyzer. Based on lowest droplet size(29.1 nm), lowest PI(0.026), lowest viscosity $(34.6 \mathrm{cp})$, optimal ZP $(-31.4 \mathrm{mV})$, highest\% $\mathrm{T}(99.4 \%)$ and lowest concentration of Triacetin $(8 \% \mathrm{w} / \mathrm{w})$, CEO nanoemulsion $\mathrm{F} 1$ was selected as a test formulation to investigate its antibacterial effects. The standard amikacin and normal saline were selected as positive and negative control, respectively. Test formulation F1 showed good inhibition zones against all bacterial strains investigated. It was observed that the inhibition zone (mm) of CEO nanoemulsion(F1) was larger than that of pure oil group against all bacterial strains. However, the inhibition zones of F1 were comparable with that of positive control (amikacin) against all bacterial strains. The antibacterial effects of F1 were superior than positive control against Staphyloccocus aureus, Proteus vulgaris and Pseudomonas aeruginosa (Table 3). The inhibition effects of pure oil group were very low against all bacterial strains as compared with F1 and positive control. On the other hand, nanoemulsion formulation without CEO (blank formulation) showed negligible zone of inhibition against all bacterial strains. The MICs of F1 were found to be lower $(0.075$ $0.085 \%$ w/w) against Bacillus subtilis, Staphyloccocus

Table 3 The antibacterial effects and minimum inhibitory concentrations of optimized clove essential oil nanoemulsion F1, pure and standard amikacin against different bacterial strains.

\begin{tabular}{lcrrrrrrrrc}
\hline \multirow{2}{*}{ Samples } & \multicolumn{4}{c}{ Inhibition zone $(\mathrm{mm})$} & \multicolumn{4}{c}{ MIC $(\% \mathrm{w} / \mathrm{w})^{\mathrm{c}}$} \\
\cline { 2 - 11 } & \multicolumn{1}{c}{ BS } & \multicolumn{1}{c}{ SA } & \multicolumn{1}{c}{ PV } & \multicolumn{1}{c}{ PA } & \multicolumn{1}{c}{ KP } & BS & SA & PV & PA & KP \\
\hline F1\# & $18.0 \pm 1.3^{*}$ & $17.0 \pm 1.2$ & $20.0 \pm 1.5$ & $16.0 \pm 1.0$ & $18.0 \pm 1.4$ & 0.080 & 0.075 & 0.085 & 0.300 & 0.250 \\
Pure CEO $^{\mathrm{a}}$ & $12.0 \pm 0.6$ & $10.0 \pm 0.4$ & $13.0 \pm 0.8$ & $9.0 \pm 0.3$ & $11.0 \pm 0.7$ & 0.130 & 0.130 & 0.130 & 0.500 & 0.400 \\
Amikacin $^{\mathrm{b}}$ & $22.0 \pm 2.0$ & $16.0 \pm 1.3$ & $15.0 \pm 0.9$ & $14.0 \pm 0.8$ & $20.0 \pm 1.6$ & 4.000 & 16.000 & 2.000 & 8.000 & 2.000 \\
\hline
\end{tabular}

Bacillus subtilis (BS), Staphyloccocus aureus (SA), Proteus vulgaris (PV), Pseudomonas aeruginosa (PA), Klebseilla pneumoniae (KP), clove essential oil (CEO), *standard deviation, sterile normal solution $(0.9 \% \mathrm{w} / \mathrm{v})$ was used as negative control (a), amikacin was used as positive control (b), \#the zones of inhibition of blank nanoemulsion (without CEO) were subtracted from zones from CEO loaded nanoemulsion, minimum inhibitory concentration (MIC), MICs of amikacin were expressed as $\mu \mathrm{g} / \mathrm{ml}$ (c) 
aureus and Proteus vulgaris as compared to Pseudomonas aeruginosa (MIC $0.300 \% \mathrm{w} / \mathrm{w}$ ) and Klebseilla pneumoniae (MIC $0.250 \% \mathrm{w} / \mathrm{w}$ ). However, the MICs of pure oil group were very low as compared to $\mathrm{F} 1$ against all bacterial strains investigated. The MICs of positive control were observed in the range of $2-16 \mu \mathrm{g} / \mathrm{ml}$ against all bacterial strains which were similar to the reported MICs of amikacin against these bacterial strains ${ }^{28-31)}$. Finally, it was observed that there was a significant difference in inhibition activity of F1 and pure oil group $(p<0.05)$. Results of ANOVA indicated that developed nanoemulsion (F1) showed significant zone of inhibition against all bacterial strains as compared to pure oil group $(p<0.05)$.

\section{CONCLUSIONS}

In the present study, nanoemulsion formulations of CEO were developed and evaluated for antibacterial effects. CEO nanoemulsions were prepared by low energy emulsification method and characterized for droplet size, PI, viscosity, ZP and transmittance. Based on lowest droplet size (29.1 nm), lowest PI (0.026), lowest viscosity (34.6 cp), optimal ZP (-31.4 mV), highest\% T (99.4\%) and lowest concentration of Triacetin ( $8 \% \mathrm{w} / \mathrm{w})$, CEO nanoemulsion F1 (containing $1 \% \mathrm{w} / \mathrm{w}$ of CEO, $8 \% \mathrm{w} / \mathrm{w}$ of Triacetin, $15 \%$ w/w of Tween-80, $15 \% \mathrm{w} / \mathrm{w}$ of Labrasol and $61 \% \mathrm{w} / \mathrm{w}$ of water) was optimized as an effective formulation of CEO and subjected to antibacterial studies. The antibacterial effects of F1 were found to be superior over pure oil and highly comparable with standard amikacin against all bacterial strains investigated. These results indicated the potential of nanoemulsions for enhancing the therapeutic efficacy of natural active ingredients such as CEO.

\section{ACKNOWLEDGEMENT}

The authors are thankful to Department of Pharmaceutics, Salman Bin Abdulaziz University, Al-Kharj, Saudi Arabia for funding and support. The authors are also thankful to Gattefosse (France) for donating the gift sample of Labrasol.

\section{CONFLICT OF INTEREST}

The authors declare no conflict of interest. The authors alone are responsible for content and writing of the paper.

\section{REFERENCES}

1) Chami, F.; Chami, N.; Bennis, S.; Bouchikhi, T.; Rem- mal, A. Oregano and clove essential oils induce surface alteration of Saccharomyces cerevisiae. Phytother. Res. 19, 405-408(2005).

2) Chaieb, K.; Hajlaoui, H.; Zmantar, T.; Kahla-Nakbi, A. B.; Rouabhia, M.; Mahdouani, K.; Bakhrouf, A. The chemical composition and biological activity of clove essential oil, Eugenia caryophyllata (Syzygium aromaticum L. Myrtaceae): a short review. Phytother. Res. 21, 501-506 (2007).

3) Fu, Y.; Zu, Y.; Chen, L.; Shi, X.; Wang, Z.; Sun, S.; Efferth, T. Antimicrobial activity of clove and rosemary essential oils alone and in combination. Phytother. Res. 21, 989-994(2007).

4) Cai, L.; Wu, C. D. Compounds from Syzygium aromaticum possessing growth inhibitory activity against oral pathogens. J. Nat. Prod. 59, 987-990 (1996).

5) Zheng, G. Q.; Kenney, P. M.; Lam, L. K. T. Sesquiterpenes from clove(Eugenia caryophyllata). J. Nat. Prod. 55, 999-1003 (1992).

6) Beuchat, L. R. Control of foodborne pathogens and spoilage microorganisms by naturally occurring antimicrobials, In Microbial Food Contamination, Wilson, C. L., Droby, S., (eds). CRC Press: Boca Raton, FL, 149-169 (2000).

7) Miyazawa, M.; Hisama M. Suppression of chemical mutagen induced SOS response by alkylphenols from clove (Syzygium aromaticum) in Salmonella typhymurium TA1535/pSK1002 umu test. J. Agr. Food Chem. 49, 4019-4025 (2001).

8) Friedman, M.; Henika, P. R.; Mandrell, R. E. Bactericidal activities of plant essential oils and some of their isolated constituents against Campylobacter jejuni, Escherichia coli, Listeria monocytogenes and Salmonella enteric. J. Food Prot. 65, 1545-1560 (2002).

9) Cressy, H. K.; Jerrett, A. R.; Osborne, C. M.; Bremer, P. $\mathrm{J}$. A novel method for the reduction of numbers of Listeriamonocytogenes cells by freezing in combination with an essential oil in bacteriological media. J. Food Prot. 66, 390-395 (2003).

10) Kalemba, D.; Kunicka, A. Antibacterial and antifungal properties of essential oils. Curr. Med. Chem. 10, 813829 (2003).

11) Ogata, M.; Hoshi, M.; Urano, S.; Endo, T. Antioxidant activity of eugenol and related monomeric and dimeric compounds. Chem. Pharm. Bull. 48, 1467-1469 (2000).

12) Park, I. K.; Lee, H. S.; Lee, S. G.; Park, J. D.; Ahn, Y. J. Insecticidal and fumigant activities of Cinnamomum cassia bark-derived materials against Mechoris ursulus (Coleoptera: Attelabidae). J. Agr. Food Chem. 48, 2528-2531 (2000).

13) Shafiq, S.; Shakeel, F.; Talegaonkar, S.; Ahmad, F. J.; Khar, R. K.; Ali, M. Development and bioavailability assessment of ramipril nanoemulsion formulation. Eur. 
J. Pharm. Biopharm. 66, 227-243(2007).

14) Shakeel, F.; Baboota, S.; Ahuja, A.; Ali, J.; Aqil, M.; Shafiq, S. Nanoemulsions as vehicles for transdermal delivery of aceclofenac. AAPS Pharm. Sci. Tech. 8, E104(2007).

15) Sakeena, M. H. F.; Elrsahid, S. M.; Munawar, A. S.; Azmin, M. N. Effects of oils and drug concentrations on droplet size of palm oil esters (POEs) nanoemulsion. $J$. Oleo Sci. 60, 155-158(2011).

16) Sakeena, M. H. F.; Yam, M. F.; Elrsahid, S. M.; Munawar, A. S.; Azmin, M. N. Anti-inflammatory and analgesic effects of ketoprofen in palm oil esters nanoemulsion. J. Oleo Sci. 59, 667-671(2010).

17) Hsieh, C. W.; Li, P. H.; Lu, I. C.; Wang, T. H. Preparing glabridin-in-water nanoemulsions by high pressure homogenization with response surface methology. $J$. Oleo Sci. 61, 483-489(2012).

18) Li, Y.; Zheng, J.; Xiao, H.; McClements, D. J. Nanoemulsion-based drug delivery systems for poorly-water soluble bioactive compounds: influence of formulation parameters on polymethoxyflavone crystallization. Food Hydrocoll. 27, 517-528 (2012).

19) Toroncoso, E.; Aguilera, J. M.; McClements, D. J. Fabrication, characterization and lipase digestibility of food-grade nanoemulsions. Food Hydrocoll. 27, 355363(2012).

20) Maswal, M.; Dar, A. H. Formulation challenges in encapsulation and delivery of citral for improved food quality. Food Hydrocoll. doi: 10.1016/j.foodhyd.2013. 10.035 .

21) Salvia-Trujillo, L.; Rojas-Grau, M. J.; Solvia-Fortuny, R.; Martin-Belloso, O. Effect of processing parameters on physicochemical characteristics of microfluidized lemongrass essential oil-alginate nanoemulsions. Food Hydrocoll. 30, 401-407 (2013).

22) Guenette, S. A.; Ross, A.; Marier, J. F.; Beaudry, F.; Vachon, P. Pharmacokinetics of eugenol and its effects on thermal hypersensitivity in rats. Eur. J. Pharmacol. 562, 60-67 (2007).

23) Jadhav, B. K.; Khandelwal, K. R.; Ketkar, A. R.; Pisal, S. S. Formulation and evaluation of mucoadhesive tablets containing eugenol for the treatment of periodontal diseases. Drug Develop. Ind. Pharm. 30, 195-203 (2004).

24） Choi, H. Y.; Yang, Y. C.; Lee, S. H.; Clark, J. M.; Ahn, Y. J. Efficacy of spray formulations containing binary mixtures of clove and eucalyptus oils against susceptible and pyrethroid/melathion-resistant head lice(Anoplu- ra: Pediculidae). J. Med. Entomol. 47, 387-391 (2010).

25) Parhi, R.; Suresh, P.; Mondal, S.; Kumar, P. M. Novel penetration enhancers for skin applications: a review. Curr. Drug Deliv. 9, 219-230 (2012).

26) Shen, Q.; Wang, Y.; Zhang, Y. Improvement of colchicines bioavailability by incorporating eugenol in the nanoemulsion as an oil excipient and enhancer. Int. $J$. Nanomed. 6, 1237-1243 (2011).

27) Nirmala, M. J.; Shivashankar, M.; Ernest, V.; Mukherjee, A.; Chandrasekaran, N. Physico-chemical characterization of ramipril using clove oil based microemulsion drug delivery system. Nanomed. Nanobiol. 1, $1-8(2013)$.

28) Alhambra, A.; Cuadros, J. A.; Cacho, J.; Gomez-Garces, J. L.; Alos, J. I. In vitro susceptibility of recent antibiotic-resistant urinary pathogens to ertapenem and 12 other antibiotics. J. Antimic. Chemother. 53, 10901094 (2004).

29) Xiong, Y. Q.; Kupferwasser, L. I.; Zack, P. M.; Bayer, A. S. Comparative efficacies of liposomal amikacin (mikasome) plus oxacillin versus conventional amikacin plus oxacillin in experimental endocarditis induced by Staphylococcus aureus: microbiological and endocardiographic analyses. Antimic. Agents Chemother. 43, 1737-1742 (1999).

30) Shrivastava, S. M.; Chaudhary, M. Comparative studies on susceptibility and minimum inhibitory concentration of potentox, a fixed dose combination of cefepime amikacin in Proteus vulgaris, Escherichia coli and Bacillus subtilis. J. Med. Sci. 9, 245-248(2009).

31) Ghaffari, S.; Varshosaz, J.; Saadat, A.; Atyabi, F. Stability and antimicrobial effect of amikacin-loaded solid lipid nanoparticles. Int. J. Nanomed. 6, 35-43(2011).

32) Shakeel, F.; Haq, N.; Elbadry, M.; Alanazi, F. K.; Alsarra, I. A. Ultra fine super self-nanoemulsifying drug delivery system (SNEDDS) enhanced solubility and dissolution of indomethacin. J. Mol. Liq. 180, 89-94 (2013).

33) Shakeel, F.; Haq, N.; Alanazi, F. K.; Alsarra, I. A. Impact of various nonionic surfactants on self-nanoemulsification efficiency of two grades of Capryol (Capryol-90 and Capryol-PGMC). J. Mol. Liq. 182, 57-63 (2013).

34) Yu, H.; Huang, Q. Improving the oral bioavailability of curcumin using novel organogel-based nanoemulsions. J. Agr. Food Chem. 60, 5373-5379(2012). 